\title{
Kongenital katarakt vaka serisi
}

\section{Congenital cataract case series}

\begin{tabular}{|c|c|c|}
\hline Ebru Demet Aygıt & Nilay Kandemir Beşek & Ceren Gürez \\
\hline Burçin Kepez Yıldız & İhsan ÇakıriD & Korhan FazıID \\
\hline Ali Demircan (iD & Birsen Gökyiğit (DD & Alper Ağca (D) \\
\hline
\end{tabular}

Sağlık Bilimleri Üniversitesi Beyoğlu Göz Eğitim ve Araştırma Hastanesi, Şaşılık Birimi, İstanbul, Türkiye

\section{ÖZ}

Amaç: Bu çalışmamızda konjenital katarakt nedeniyle hastanemize başvuran hastaların başvuru yaşı, operasyon zamanı, uygulanan cerrahi teknik ve intraoküler lens implantasyonu, posterior kapsül kesafeti ve elde edilen görsel sonuçların değerlendirilmesi amaçlanmıştır.

Gereç ve Yöntem: Hastanemizde, 2016-2017 yılları arasında konjenital katarakt cerrahisi geçirmiş hasta dosyaları retrospektif olarak incelenmiştir. Çalışmamıza 25 (16 erkek, 9 kız) hastanın 42 (24 sağ, 18 sol) gözü dahil edilmiştir. Altı aydan kısa takip süresi olan hastalar çalışmaya dahil edilmemiştir. Vakaların demografik özellikleri, elde edilen en iyi düzeltilmiş görme keskinlikleri ve ön segment bulguları kaydedilmiştir. İstatistiksel analizler SPSS programı ile yapılmıştır.

Bulgular: Yaş ortalaması 6,5 \pm 3 yıl (3-12) ve ortalama takip süreleri 2,7 yıl (2-3,5) idi. Bir yıllık süre içinde 17 hastaya bilateral, sekiz hastaya unilateral konjenital katarakt cerrahisi yapılmıştır. Tüm hastalara ko-aksiyel irrigasyon aspirasyon ile lens aspirasyonu, primer posterior kapsüloreksis, anterior vitrektomi ve intraoküler lens implantasyonu uygulanmıştır. Takiplerde 23 hastada ambliyopi tespit edilmiştir.

Sonuç: Kongenital katarakt gelişmekte olan ve az gelişmiş ülkelerde çocukluk çağı körlüklerinin önemli bir nedenidir. Bu nedenle görme gelişimi açısından konjenital katarakt hastalarının tespit edilmesi, cerrahi zamanlama, cerrahi teknik ve hastaların düzenli takiplerinin yapılması önemlidir.

Anahtar Sözcükler: Konjenital katarakt, az görme, ambliyopi.

\section{ABSTRACT}

Aim: To evaluate admitted age, operation time, surgical technique, intraocular lens implantation, posterior capsule opacification and visual results in a series of cases with congenital cataract.

Materials and Methods: The patients who underwent congenital cataract surgery between 2016-2017 in our hospital were retrospectively evaluated. 42 (24 right, 18 left) eyes of 16 boys, and 9 girls were included in this study. Patients with less than six months of follow-up time were not included. Demographic features of patients, best corrected visual acuity, anterior segment findings were recorded. Statistical analysis was performed with SPSS program.

Results: The mean age was 6,5 3 years (3 - 12) and the mean follow-up period was 2,7 years (2 3.5). Eight unilateral and 17 bilateral congenital cataract surgeries were performed within a year. All patients underwent lens aspiration with co-axial irrigation-aspiration, primary posterior capsulorhexis, anterior vitrectomy and intraocular lens implantation. In the follow-up, amblyopia was detected in 23 patients.

Sorumlu yazar: Ebru Demet Aygıt

Sağlık Bilimleri Üniversitesi Beyoğlu Göz Eğitim ve Araştırma

Hastanesi, Şaşıık Birimi, İstanbul, Türkiye

E-posta: ebrudemet@hotmail.com

Başvuru tarihi: 28.07.2020 Kabul tarihi: 19.02.2021 
Conclusion: Congenital cataract is an important cause of childhood blindness in developing and under-developed countries. Thus, early detection of congenital cataract patients, and surgical timing, surgical technique and regular follow-up are important in terms of the development of visual functions.

Keywords: Congenital cataract, low vision, ambliopia.

\section{GíRiş}

Katarakt dünya genelinde önlenebilir ve tedavi edilebilir körlük nedenlerinin başında gelmektedir. Dünya Sağlık Örgütü (DSÖ) ve Uluslararası Körlüğü Önleme Ajansı'nın (IAPB) 1999 yılında ortaya koydukları temel amaçlarını "2020 yılına kadar önlenebilir körlüğü ortadan kaldırmak ve önlenebilir görsel bozuklukların artmasını engellemektir" şeklinde açıklamışlardır (1). Foster ve ark. çalışmalarına göre, dünya genelinde katarakta bağlı körlük yaşayan çocuk sayısı yaklaşık olarak 200.000 civarındadır (2). Çocukluk çağı katarakt prevalansını Sheeladevi ve ark. çalışmalarında $1,03 / 10.000$ olarak bildirmiştir (3).

Konjenital kataraktı olan hastalarda, görme fonksiyon kaybının major nedeni deprivasyon ambliyopisidir. Tek taraflı görme deprivasyonu yapılmış hayvan deneylerinde, erken kritik dönemde oluşan değişikliklerin geri döndürülebildiği gösterilmiştir. Doğumdan sonraki ilk 6-8. haftalarda opere edilen tek taraflı konjenital katarakt hastalarında, optik düzeltme ve kapama tedavisi ile normale yakın görme keskinliği, füzyon ve stereopsis gelişimi sağlanabildiği saptanmıştır (4-7).

Biz de çalışmamızda konjenital katarakt cerrahisi uygulanan hastalarımızın demografik özelliklerini, takip ve tedavi sonuçlarımızı değerlendirmeyi amaçladık.

\section{GEREÇ ve YÖNTEM}

Beyoğlu Göz Eğitim ve Araştırma Hastanesinde, 2016-2017 yılları arasında Ön segment ve Şaşılık birimlerinde, konjenital katarakt nedeniyle takip ve tedavileri yapılan hastalarımızın kayıtları incelenmiştir. Hayatının ilk yılı içerisinde gelişmiş kataraktı olan hastalar çalışmaya dahil edilmiştir. Altı ay altında takibi olan hastalar çalışma dışında bırakılmıştır. Düzenli takiplerine gelebilen ve kayıtlarına ulaşılabilen 25 hastanın 42 gözü çalışmamıza dahil edilmiştir. Hastalarımızın yaş ortalaması 6,5 \pm 3 yıldır (3-12 yıl). Hastaların demografik özellikleri, operasyon özellikleri ve postoperatif sonuçları dosyalar üzerinden retrospektif olarak incelenmiştir. Hastanemiz lokal etik komitesinden çalışma onayı alınmıştır
(Beyoğlu Göz Eğitim ve Araştırma Hastanesi, 31.08.2018, 18/H-1). Çalışmamız Helsinki Protokolüne uygun şekilde yürütülmüştür.

İlk başvuru sırasında hastaların detaylı oftalmolojik muayeneleri yapılmıştır. Belirgin şaşılığı olan hastalar şaşılık biriminde de değerlendirilmiştir. Hastaların refraktif durumlarını belirlemede manuel otorefraktometre (Righton, Retinomax-K plus 3, Nikon Inc, Melville, NY, USA) ölçümleri, yaşlarına uygun olarak siklopentalat hidroklorid (Sikloplejin 1\%, Abdi İbrahim İlaç San. ve Tic. A.Ş.) göz damlasının 2$3 \mathrm{kez}, 5$ dakika ara ile uygulanması sonrasında yapılmıştır.

Hastaların görme keskinlikleri Lea Gratings $(0,25$ CPCM - 8,0 CPCM, Licensed by Lea-Test Ltd.) veya Snellen resimli çocuk eşeli ile ölçülmüştür. Şaşlık muayenesinde Hircshberg testi, kapama testi, prizma kapama testi veya Krimsky testi ve 9 diagnostik bakış pozisyonunda göz hareketleri değerlendirmesi yapılmıştır.

\section{Cerrahi teknik}

Operasyonlar aynı cerrah tarafından, genel anestezi altında ve CENTURION ${ }^{\circledR}$ Vision System (Alcon Laboratories, Inc., Fort Woth, TX, USA) fakoemülsifikasyon cihazı ile yapıldı. 2-4 mm temporal şeffaf korneal ana insizyon ve iki adet yan giriş yapıldı. Ön kamara dispersif viskoelastik madde (VEM) (Viscoat ${ }^{\circledR}$, Alcon Laboratories, Inc., Fort Woth, TX, USA) ve kohezif VEM (Provisc ${ }^{\circledR}$, Alcon Laboratories, Inc., Fort Woth, TX, USA) verilerek, soft shell tekniği ile dolduruldu. Yaklaşık 5,5 mm'lik anterior curvelineer kapsüloreksis yapıldıktan sonra lens materyali 0,5 mm'lik açıklığa sahip ko-aksiyel irrigasyon aspirasyon (I/A) probu ile alındı. Arka kapsul insülin enjektörü ile açıldı ve bu açıklıktan ön hyaloid ve arka kapsül arasına dispersif VEM verildi. Yaklaşık $5 \mathrm{~mm}$ 'lik primer posterior curvelineer kapsüloreksis (PPCK) ardından bir miktar anterior vitrektomi yapıldı. Kese içi kohesif VEM ile dolduruldu. 2-4 mm'lik insizyon genişletilmeden, enjektörle kese içine mono-blok, hidrofobik akrilik intraoküler lens (IOL) implante edildi. Lens optiğinin arka kapsül arkasına alınması yani posterior optik yakalama uygulandı. Ön kamarada kalmış olan VEM I/A probu ile 
temizlendi. Yara yerleri hidrate edildikten sonra sızdırmazlık kontrolü yapıldı ve gereken durumlarda 10.0 naylon ile sütüre edildi. Ön kamaraya $0,1 \mathrm{ml}$ triamsinolon asetonid (Kenacort-A, DEVA, İstanbul, Türkiye) ile subkonjonktival atropin (Atropin Sülfat, Galen İlaç, İstanbul, Türkiye) ve steroid (Dekort ampul, DEVA, İstanbul, Türkiye) yapılarak ameliyat sonlandırıldı. Ameliyat sonrası birinci ayda genel anestezi altında muayene ve sütür alımı yapıldı. Postoperatif erken dönemde steroid göz damlası 12x1 başlandı, inflamasyon durumuna göre ilk haftadan itibaren kademeli olarak $6 \times 1$ azaltarak $1-1,5$ ay içinde tamamen kesildi, antibiyotik $4 \times 1$ ve 5 gün kullanıldı. Birinci ay, 3. ay ve 6 . ayda takip muayeneleri yapıldı.

Katarakt cerrahisi sonrasında, postoperatif ilk hafta içinde özellikle lens implantasyonu yapılmamış olan hastalar değerlendirilmiş, gerekli refraktif düzeltmeleri (kontakt lens $(\mathrm{KL})$ veya gözlük camı) yapılmıştır. Ambliyopi tespit edilen hastalarda, gerekli kapama tedavisi 2/4/6 saat olarak düzenlenmiştir.
İstatistiksel analizler SPSS programı (14.0 for windows) ile yapılarak, $p$ değerinin $\leq 0,05$ olması anlamlı kabul edilmiştir. Sıklık ve tanımlayıcı analiz testleri kullanılmıştır.

\section{BULGULAR}

Hastaların demografik özellikleri Tablo-1a ve 1 b'de gösterilmiştir. Hastanın ilk muayenesi ve cerrahiye karar verilmesi ile cerrahi yapılması arasında geçen süre ortalaması $35,9 \pm 25,6$ gündür (4-90 gün). Hastalarımızın ortalama takip

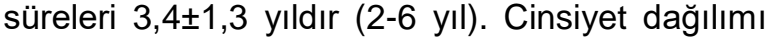
açısından bakıldığında; Kız/erkek çocuk dağılımı \%36 kız çocuk ve \%64 erkek çocuk şeklindedir.

Hastalarımızın preoperatif sferik ekivalan (SE) değerleri $-12,50$ ile $+6,00$ D (ortalama $-0,74,7$ ) arasında değişmektedir. Preoperatif görme keskinlikleri kooperasyon kısıtııığı nedeniyle her hastadan alınamamıştır. Görme keskinliği değerlendirilebilen hastalarda preoperatif en iyi düzeltilmiş görme keskinlikleri (EIDGK) ortalama $0,3 \pm 0,2$ 'dir (en az 0,05 ile en çok 0,7). Postoperatif elde edilen SE değeri ortalaması 2,05 1,9 D'dir (-6,50 D ile +1,25 D).

Tablo-1a. Hastalarımızın demografik özellikleri.

\begin{tabular}{ccccccccc}
\hline \multirow{2}{*}{ Toplam Hasta Sayısı } & \multicolumn{2}{c}{ Cinsiyet } & \multicolumn{2}{c}{ Taraf } & \multicolumn{2}{c}{ Lateralite } & \multicolumn{2}{c}{ Yaş ortalaması } \\
& erkek & kız & sağ & sol & unilateral & bilateral & (yıl) \\
\hline 25 & 16 & 9 & 24 & 18 & 8 & 17 & $6,7 \pm 2,3(3-12)$ \\
\hline
\end{tabular}

Tablo-1b. Hastalarımızın demografik özellikleri.

\begin{tabular}{llllll}
\hline Yaş Grupları & Hasta Sayısı & EiDGK & Sferik Ekivalan (D) & Şaşılık & Ambliyopi \\
\hline 3-5 yaş & 7 & $0,85 \pm 0,2$ & $-2,0 \pm 2,6$ & $5(\% 71,4)$ & $7(\% 100)$ \\
5-8 yaş & 10 & $1,0 \pm 0,3$ & $-2,8 \pm 2,1$ & $5(\% 50)$ & $10(\% 100)$ \\
$8-12$ yaş & 8 & $0,52 \pm 0,5$ & $-1,4 \pm 0,99$ & 0 & $5(\% 62,5)$ \\
\hline
\end{tabular}

EIDGK: En İyi Düzeltilmiş Görme Keskinliği, D: Dioptri

Tablo-2. Görülen katarakt tipleri.

\begin{tabular}{ll}
\hline Katarakt Tipi & Hasta Sayısı \\
\hline Nükleer Katarakt & $15(\% 35,7)$ \\
Lameller Katarakt & $13(\% 31)$ \\
Polar Katarakt & $8(\% 19)$ \\
Kapsüler Katarakt & $6(\% 14,3)$ \\
\hline
\end{tabular}


Postoperatif elde edilen EIDGK ortalama $0,5 \pm$ 0,2 'dir (en az 0,1 ile en çok 1,0). Preoperatif ve postoperatif EIDGK arasında istatistiksel olarak anlamlı farklılık bulunmaktadır $(p \leq 0,001)$.

Hastalarımızı yaşlarına göre (3-5 yaş, $5-8$ yaş ve 8-12 yaş) gruplandırıp, postoperatif EIDGK, SE, şaşılık ve ambliyopi sonuçları gruplar arasında karşılaştırılmıştır. EIDGK ve SE değerlerinde gruplar arasında farklılık tespit edilmemiştir. Şaşılık ve ambliyopi açısından bakıldığında ise gruplar arasında istatistiksel olarak anlamlı farklılık tespit edilmiştir (ANOVA, sırasıyla $\mathrm{p}<$ $0,001, p<0,001$ ).

Postoperatif takip döneminde, $15 \quad(\% 35,7)$ hastada şaşılık, $38 \quad(\% 90,5)$ gözde ambliyopi tespit edilmiş, preoperatif 15 hastada görülen nistagmus postoperatif $11 \quad(\% 26,2)$ hastada görülmüş, dört hastada düzelme sağlanmıştır. Takip sırasında hastalarımızın hiçbirinde glokom saptanmamıştır.

Hastalarımızda en sık görülen katarakt tipi \%35,7 oranı ile nükleer katarakttır. Nükleer kataraktı sırasıyla lameller, polar ve kapsüler katarakt takip etmektedir (Tablo-2). Uygulanan cerrahi lens aspirasyonu + PPCK + Anterior vitrektomi + IOL implantasyonudur. Postoperatif takip döneminde $12(\% 28,6)$ gözde posterior kapsül kesafeti PKK gelişmiştir.

Lateralite değerlendirildiğinde, çalışmamızda 17 (\%68) hastada bilateral konjenital katarakt görülmüştür.

On bir $(\% 26,2)$ hastanın anamnezinde doğuştan katarakt açısından pozitif aile öyküsü bulunmaktadır. Ek sistemik patoloji dört $(\% 9,5)$ hastada, ek oküler patoloji yedi $(\% 16,7)$ hastada tespit edilmiştir. Dört (\%16) hastada geçirilmiş konjenital enfeksiyon tanısı bulunmuştur.

\section{TARTIŞMA}

Dünya genelinde çocukluk çağı (0-15 yaş arası) ağır görme bozukluğu ve körlük prevalansı, zengin ülkelerde 0,1/1000 iken fakir ülkelerde yaklaşık 1,1/1000 olarak bildirilmiştir (4). Konjenital katarakt, çocukluk çağı önlenebilir körlük sebeplerinin başında gelmektedir. Tabin ve ark. çalışmalarında, gelişmekte olan ülkelerde çocukluk çağı körlüklerinin \%75'inin konjenital katarakta bağlı olduğu gösterilmiştir (6). Fransa'da konjenital katarakt cerrahisi oranı 1$3 / 10,000$ doğum olarak gösterilmiş ve bu oranın gelişmiş ülkelerdeki oranla uyumlu olduğu bildirilmiştir (8).

Konjenital kataraktlı hastaların en iyi katarakt ekstraksiyon zamanı halen cerrahlar arasında tartışma konusu olmaya devam etmektedir (17, 18). Yapılan çalışmalarda dikkat çekilen ortak nokta, görsel gelişim için cerrahi zamanlamanın kritik rol oynadığıdır. Hastaların uygun zaman ve uygun cerrahi ile takip ve tedavileri yapılmaz ise görme fonksiyonu ve gözün gelişimi açısında geri döndürülemez sonuçlar gelişebileceği belirtilmektedir (19). Hayatın ilk sekiz haftası içinde yapılan katarakt cerrahisi ile daha iyi sonuçlar alındığı çalışmalarda bildirilmiştir (20, 21). Maurer ve Lewis, 5 aylık iken ameliyat ettikleri hastalarını 3 yaş ve üzerinde değerlendirmişlerdir. Sonuç görme keskinliklerini ortalama $20 / 80$ olarak tespit etmişler ve cerrahi yaşı ile görme keskinliği arasında doğrusal bir korelasyon tespit etmişlerdir (22).

Literatürde, konjenital katarakt cerrahisinin geciktirilmesinin tedaviye dirençli ambliyopi riskini artırdığını ve potansiyel görsel fonksiyonları azalttığını gösteren birçok çalışma bulunmaktadır $(23,24)$.

Birch ve ark. çalışmalarında; risklerine rağmen hayatın ilk dört haftasında katarakt cerrahisi uyguladıkları hastalarında, uzun dönem görme keskinliği açısından mükemmel sonuçlar elde ettiklerini bildirilmişlerdir. Yoğun kataraktı olan hastalarda, erken katarakt cerrahisinin risklerine rağmen, deprivasyon ambliyopisini en aza indirebileceği sonucunu açıklamışlardır (23). Konjenital katarakt cerrahisi uygulanma yaşları yayınlarda 3,5 ile 7 yıl arasında bildirilmiştir (2527). Bizim çalışmamızda ortalama konjenital katarakt cerrahi uygulama yaşı 6,6 $\pm 2,3$ yıl olarak tespit edilmiştir. Bu sürenin geç olması hastaların bize geç refere edilmesi ile ilişkili olabilir. Çünkü bizim hastayı değerlendirip cerrahi kararı vermemiz ve cerrahi uygulamamız arasında

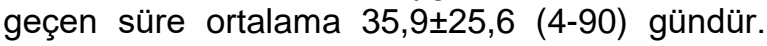
Ayrıca hastanemiz dal hastanesi olması ve sınırlı imkanları, konjenital kataraktı olan hastalara çok erken dönemde cerrahi uygulanamamasının diğer bir olası nedenidir.

Glokom, konjenital katarakt cerrahisinin korkulan bir komplikasyonudur. Postoperatif glokom gelişim oranı literatürde \%8 ile \%59 arasında bildirilmiştir (28-36). Erken cerrahinin avantajları yanında, postoperatif glokom gelişiminde risk faktörü olduğu yayınlarla gösterilmiştir $(37,38)$. The Infant Aphakia Treatment Study (IATS) çalışmasında, beş yıllık takipte, postoperatif glokom insidensi IOL implantasyonu yapılan grupta \%18, IOL implantasyonu yapılmayan grupta \%16 olarak verilmiştir (39). Viswanath ve ark.'nın beş yıl takip süreli 128 olgudan oluşan serisinde, hayatın ilk 4 haftasında ameliyat olan 
gözlerde glokom oranı \%31,6, dördüncü haftadan sonra ameliyat olan gözlerde glokom oranı \%9,3 olarak bildirilmiştir (37). Glokom gelişimi için önemli iki risk faktörü olarak cerrahi uygulanma yaşının küçük olması ve cerrahi uygulandığı sırada daha küçük kornea çapına sahip olunması gösterilmiştir (39). Bizim çalışmamızda katarakt cerrahisi uygulanma yaşının nispeten geç olması sebebi ile hastalarımızda postoperatif glokom gelişmediği düşünülmektedir.

Katarakt cerrahisi sonrasında afakinin düzeltilmesinde; primer IOL implantasyonu, gözlük/KL kullanımı ve sekonder IOL implantasyonu seçenekleri bulunmaktadır. Günümüzde, gelişen teknoloji ve kullanılan malzemelerde sağlanan gelişmeler ile 2 yaş ve üzerindeki hastalarda katarakt cerrahisi ve IOL implantasyonunun aynı seansta yapılması önerilmektedir. İki yaş altındaki hastalarda ise tartışmalar devam etmektedir (40). Çalışmamızda hastalarımıza katarakt cerrahisi sırasında primer IOL implantasyonu yapılmış, hastalar afak bırakılmamıştır.

Aynı seansda IOL uygulanmasının infant gözlerinde uzun dönemde güvenilirliği halen cerrahlar arasında tartışmalı bir konudur. Bu konuyu aydınlatmak için IATS bir çalışma yapmıştır (41). Çalışmanın birinci yıl sonuçlarında, cerrahi sırasında IOL implantasyonu uygulanan ve uygulanmayan hastalar arasında istatistiksel açıdan farklılık görülmemiştir. Dikkat çeken bir özellik ise IOL implantasyonu yapılan grupta, ek göz içi ameliyat oranlarında belirgin artış (IOL grubunda \%18, IOL uygulanmayan grupta \%4) tespit edilmiş olmasıdır (42). Beşinci yılda, IOL implantasyonu yapılan gruptaki hastaların çoğunda en az bir ek durum, sıklıkla lens reproliferasyonu, pupillar membranlar ve korektopi görülmüştür. İntraoküler lens implantasyonu uygulanmayan (bu grup $\mathrm{KL}$ grubu olarak isimlendirilmektedir) gruptaki hastalarda da beşinci yıl sonuçlarında ek durumların gelişme sıklığı artış gösterse de oran IOL grubundaki hastalarda daha büyüktür (39).

İntraoküler lens implantasyonu yapılan hastalarda sıklıkla gelişen komplikasyon PKK'dir. Trivedi ve ark.'nın çalışmalarında PKK oranı \%37,9'dur (43). Negalur ve ark.'nın çalışmalarında ise PKK oranının \%18,3 olduğu ve sıklıkla postoperatif 6 ay içinde görüldüğünün altı çizilmiştir (44). PKK, IOL implantasyonu yapılan ya da yapılmayan olgularda, pediatrik katarakt cerrahisi sonrası en sık karşılasılan komplikasyondur. $\mathrm{Bu}$ nedenle primer cerrahi sırasında posterior kapsüloreksis ve anterior vitrektomi önerilmektedir $(45,46)$. Fenton ve O'Keefe (47) konjenital katarakt operasyonu sonrasında, PPCK yapılan ve anterior vitrektomi yapılmayan gözlerde PKK gelişimi oranını \%15,6 olarak bildirmişlerdir. Vasavada ve ark. çalışmalarıyla, lens epitel hücrelerinin migrasyon ve yenilenmesini, aynı zamanda anterior vitre ile IOL optiği arasındaki temasın yol açtığı vitre opafikasyonunu engellemek için rutin anterior vitrektomiyi önermiştir (48). Bizim çalışmamızda hastalarımıza PPCK ve anterior vitrektomi rutin olarak uygulanmıştır ve postoperatif takip döneminde \%28,6 oranında PKK gelişmiştir.

Çalışma grubumuzda, 6 aylık ve altında hasta bulunmamaktadır. Bu yaş grubununda cerrahi yaklaşımı değerlendiren Negalur ve ark. çalışmalarında, 6 aylık ve altında katarakt cerrahisi ile birlikte birincil IOL uygulanmasının güvenli bir şekilde yapılabileceği bildirilmiştir. Cerrahi planlanan bu yaş grubu hastalarda; anterior segment disgenezisi olmaması, konjenital glokom olmaması, kornea çapları ve aksiyel uzunluk ölçümlerinin yaş ile uyumlu olmasının kritik öneme sahip olduğu belirtilmiştir (44).

Çalışmamızda bilateral kataraktı bulunan hastalarda eş zamanlı cerrahi uygulanmamıştır. Literatürde bu konu ile ilgili değişik çalışmalar bulunmaktadır. Umar ve ark. çalışmalarında \%77 oranında eş zamanlı cerrahi uyguladıklarını bildirmişlerdir. Eş zamanlı cerrahi uygulayarak maliyet azalması, anestezi riski azalması ve ikinci gözün cerrahisini kaçırmamak gibi avantajlar elde ettiklerini belirtmişlerdir (25). Ramsay ve ark. da eş zamanlı cerrahi uyguladıkları çalışmalarında, ciddi intraoperatif ve postoperatif komplikasyonlar ile karşılaşmadıklarını ve görsel sonuçlarının iyi olduğunu göstermişlerdir (49). Totan ve ark. geniş yaş aralığına sahip hasta grubu ile yaptıkları çalışmalarında, cerrahi asepsi kurallarına sıkıca uyulduğunda, tek seansta bilateral katarakt cerrahisinin güvenli ve faydalı olduğu bildirmiştir (50). Eş zamanlı cerrahiyi öneren yazarlar tekrarlayan preoperatif muayenelerden kaçınmak, genel anestezi risklerini azaltmak, seyahat süresi ve seyahat maliyetlerini azaltmak, daha az hasta takip viziti ve daha az iş güç kaybı gibi hasta ve toplum için bazı yararlar belirtmektedirler $(51,52)$.

Dave ve ark. çalışmalarında, bilateral konjenital katarakt hastalarında eş zamanlı ve ardışık cerrahiyi karşılaştırmışlardır. Endoftalmi her iki tedavi grubunda da görülmemiştir. Endoftalmi gelişimini önleyebilmelerini her konuda fazlasıyla hassasiyet gösterilmiş olması, sterilizasyon 
kurallarına tam olarak uyulması, ikinci göze geçildiğinde gözün yeniden silinmesi ve ameliyat örtüsünün değiştirilmesi ve her göz için ayrı alet ve solüsyon kullanılmış olması ile ilişkilendirmişlerdir. Çalışmada cerrah deneyimine de özen gösterildiği bildirilmiştir. Aynı çalışmada görsel sonuçlar açısından da gruplar arasında istatistiksel olarak anlamlı farklıık bulunmamıştır (53).

Amerika Birleşik Devletleri'nde artmış hasta risklerinden dolayı (özellikle bilateral endoftalmi) eş zamanlı bilateral katarakt cerrahisi nadir olarak yapılmaktadır $(54,55)$. Literatürde, bilateral kataraktı olan çocuklarda aynı seansta cerrahi yapılmıyorsa ve ameliyat edilmeyen gözde ambliyopi yaratabilecek yoğunlukta katarakt varsa ikinci gözün operasyonu mümkün olan en erken dönemde planlanmasının gerektiği bildirilmektedir (56).

Mikrocerrahi tekniklerindeki gelişmeler, konjenital katarakt cerrahisini daha güvenli hale getirse de peroperatif ve postoperatif komplikasyon görülme sıklığı hala yüksektir (53-56). Konjenital katarakt cerrahisi yapılmasına karar verilip, cerrahi hazırlıkları başladığında hastanın karşılaşabileceği riskler gözden geçirilmelidir. Erken dönemde görülebilecek kanama, yoğun inflamasyon, enfeksiyon ve uzun dönemde görülebilecek PKK, sekonder membranlar, lens materyali reproliferasyonu, glokom, şayet yerleştirildiyse IOL dislokasyonu ve retina dekolmanı karşılaşılabilecek komplikasyonlardır. Bu durumlar ek cerrahiyi gerektirebilmektedir (5759). Postoperatif yoğun reaksiyon, komplike ve travmatik kataraktı hasta grubunda daha sık görülmektedir. Gelişen yoğun reaksiyon posterior sineşi, seklüzyo pupilla gelişimi ve sekonder açı kapanması glokomuna neden olabilmektedir. Cerrahi tekniklerin geliştirilmesi yanında postoperatif dönemde sikloplejik ajanlar ve sık steroid kullanımı ile postoperatif yoğun reaksiyon görülme sıklığında belirgin azalma sağlanabilmektedir (60). İntraoküler lens optiğinin kısmi olarak irisin anterioruna doğru yer değiştirmesi erken dönemde gelişebilen bir komplikasyondur. Gelişen pupil distorsiyonu ve fotofobi nedeniyle hastalar ciddi görme bozukluğu yaşayabilmektedir. Bu komplikasyonun, cerrahi sırasında IOL'nin dikkatli yerleştirilmesi ve optik yakalama yapılması ile azaltılabileceği yayınlarda gösterilmiştir (60-65). Katarakt cerrahisi geçiren çocuklarda yaşam boyu retina dekolmanı görülme riski artış göstermektedir. Vitreus hemorajisi de görülebilmekle birlikte, daha az geliştiği tespit edilmiştir. Retina hemorajisi, erken postoperatif dönemde karşılaşıllabilen bir diğer komplikasyondur. Operasyon sonunda düşük göz içi basıncı ile ilişkili olabileceği bildirilmiştir (63). Çalışmamızda postoperatif erken dönemde ve sonraki takiplerde herhengi bir yoğun ön kamara reaksiyonu, retina problemleri ve IOL dislokasyonu izlenmemiştir.

Çalışmamızda hastalarımızda postoperatif elde edilen EIDGK ortalama $0,5 \pm 0,2(0,1-1,0)$ ve preoperatif ve postoperatif görme değerleri arasında istatistiksel olarak anlamlı farklılık bulunmuştur. Erken cerrahi kadar, cerrahi geçirildikten sonraki tedavi ve takipler de önemlidir. Ambliyopi tedavisi amacıyla uygulanması gereken oklüzyon tedavisi ile bakıcı ve/veya ailenin eğitim ve sosyo-kültürel durumunun ilişkisi daha önce yapılmış çalışmalarla saptanmıştır (66). Aile veya bakıcının oklüzyon tedavisinin önemi konusundaki eğitimi tedavi başarısı için oldukça önemlidir $(67,68)$. Bu nedenle, hastalarımızın takiplerini Şaşılık ve Ön segment birimlerinde ve aileyi de tedavinin bir parçası haline getirerek devam ettirmekteyiz.

Çalışmamızın bazı kısıtılıkları bulunmaktadır. Bunlardan biri retrospektif bir çalışma olmasıdır. Aynı zamanda sınırlı vaka sayısı ve göreceli olarak kısa takip periyodu diğer kısıtlılıklarıdır.

\section{SONUÇ}

Çalışmamız, konjenital katarakt nedeniyle ameliyat edilen hastalarda fonksiyonel başarının anatomik başarıyla eşdeğer olmadığını bir kez daha vurgulamaktadır. Konjenital katarakt cerrahisi konusundaki deneyim ve komplikasyonsuz gerçekleştirilen cerrahi yanında, nihai başarıda kritik öneme sahip olan ameliyat zamanlaması ve ameliyat sonrası görsel rehabilitasyonun doğru ve zamanında yapılmasıdır.

Çıkar çatışması: Yazarlar çıkar çatışması beyan etmemişlerdir. 


\section{Kaynaklar}

1. WHO. Geneva: WHO; 2000. Preventing blindness in children: report of WHO/IAPB scientific meeting. Programme for prevention of blindness and deafness and International Agency for prevention of blindness. Available from: http://www.who.int/blindness/en/index.html. [Last accessed on $2011 \mathrm{Feb} 26$ ].

2. Foster A, Gilbert C. Epidemiology of childhood blindness. Eye (Lond). 1992; 6:173-6.

3. Sheeladevi S, Lawrenson JG, Fielder AR, Suttle CM. Global prevalence of childhood cataract: a systematic review. Eye (Lond). 2016; 30 (9): 1160-9.

4. Gilbert CE, Anderton L, Dandona L, Foster A. Prevalence of visual impairment in children: a review of available data. Ophthalmic Epidemiol. 1999; 6 (1): 73-82.

5. Rogers NK, Gilbert CE, Foster A, Zakhidov BO, McCollum CJ. Childhood blindness in Uzbekistan. Eye (Lond). 1999; 13: 65-70.

6. Tabin G, Chen M, Espandar L. Cataract surgery for the developing world. Curr Opin Ophthalmol. 2008; 19: 55-9.

7. Birch EE, Stager D, Leffler J, Weakley D. Early treatment of congenital unilateral cataract minimizes unequal competition. Invest Ophthalmol Vis Sci. 1998; 39 (9): 1560-6.

8. Daien V, Le Pape A, Heve D, Villain M, Bremond Gignac D; Collaborators of the Epidemiology and Safety Program (EPISAFE). Incidence and Characteristics of Congenital Cataract Surgery in France from 2010 to 2012: The EPISAFE Program. Ophthalmic Res. 2017; 58 (2): 114-6.

9. Kim KH, Ahn K, Chung ES, Chung TY. Clinical outcomes of surgical techniques in congenital cataracts. Korean J Ophthalmol. 2008 Jun; 22 (2): 87-91.

10. Zimmermann-Paiz MA, Quiroga-Reyes CR. Pediatric cataract in a developing country: retrospective review of 328 cases. Arq Bras Oftalmol. 2011; 74 (3): 163-5. Spanish.

11. Lin D, Liu Z, Chen J, Lin Z, Zhu Y, Chen C, Wu M, Lin H, Chen W, Liu Y. Practical pattern of surgical timing of childhood cataract in China: A cross-sectional database study. Int J Surg. 2019; 62: 56-61. doi: 10.1016/j.jisu.2019.01.012. Epub 2019 Jan 20.

12. Fox A, O'Keefe M, Lanigan B. A follow-on study on vision-related quality of life assessment using the NEIVFQ-25 in those with a history of unilateral and bilateral congenital cataracts. Acta Ophthalmol. 2018; 96 (5): e596-e599. doi: 10.1111/aos.13692.

13. Louison S, Blanc J, Pallot C, Alassane S, Praudel A, Bron AM, Creuzot-Garcher C. Visual outcomes and complications of congenital cataract surgery. J Fr Ophtalmol. 2019; 42 (4): 368-74. doi: 10.1016/j.jfo.2018.10.007. Epub 2019 Mar 18.

14. Mérula RV, Fernandes LC. Infantile cataract: the importance of early treatment and diagnosis. Arq Bras Oftalmol. 2005; 68 (3): 299-305. Epub 2005 Jul 26.

15. Haargaard B, Wohlfahrt J, Fledelius HC, Rosenberg T, Melbye M. Incidence and cumulative risk of childhood cataract in a cohort of 2.6 million Danish children. Invest Ophthalmol Vis Sci. 2004; 45 (5): 1316-20.

16. Nyström A., Almarzouki N., Magnusson G., Zetterberg M. Phacoemulsification and primary implantation with bag-in-the-lens intraocular lens in children with unilateral and bilateral cataract. Acta Ophthalmol. 2018; 96 (4): 364-70. doi: 10.1111/aos.13626. Epub 2018 Jan 19.

17. Birch EE, Stager DR. The critical period for surgical treatment of dense congenital unilateral cataract. Invest Ophthalmol Vis Sci. 1996; 37 (8): 1532-8.

18. Jeffrey BG, Birch EE, Stager DR, Jr., et al. Early binocular visual experience may improve binocular sensory outcomes in children after surgery for congenital unilateral cataract. Journal of AAPOS. 2001; 5 (4): 209-16.

19. Medsinge A, Nischal KK. Pediatric cataract: challenges and future directions. Clin Ophthalmol. 2015; 9: 77-90.

20. Dahan, E., Salmenson, B. - Pseudophakia In Children: Precautions, Technique, And Feasibility. J Cataract Refract Surg 1990; 16:75-82.

21. Elston, J.S., Tımms, C. - Clinical evidence for the onset of the sensitive period in infancy. $\mathrm{Br} \mathrm{J}$ Ophthalmol 1992; 73: 327-8.

22. Maurer D, Lewis TL. Visual outcomes after infantile cataract. In: Simons K, editor. Early Visual Development: Normal and Abnormal. Oxford University Press; New York: 1993. 
23. Birch EE, Wang J, Felius J, Stager DR, Jr, Hertle RW. Fixation control and eye alignment in children treated for dense congenital or developmental cataracts. J AAPOS. 2012; 16 (2): 156-60.

24. Lambert SR, Lynn MJ, Reeves R, Plager DA, Buckley EG, Wilson ME. Is there a latent period for the surgical treatment of children with dense bilateral congenital cataracts. J AAPOS. 2006; 10 (1): 30-6.

25. Umar MM, Abubakar A, Achi I, Alhassan MB, Hassan A. Pediatric cataract surgery in National Eye Centre Kaduna, Nigeria: outcome and challenges. Middle East Afr J Ophthalmol. 2015 Jan-Mar; 22 (1): 92-6.

26. Yorston D, Wood M, Foster A. Results of cataract surgery in young children in east Africa. Br J Ophthalmol. 2001; 85: 267-71.

27. Thakur J, Reddy H, Wilson ME, Jr, Paudyal G, Gurung R, Thapa S, et al. Pediatric cataract surgery in Nepal. J Cataract Refract Surg. 2004; 30: 1629-35.

28. Kuhli-Hattenbach C, Luchtenberg M, Kohnen T, Hattenbach LO. Risk factors for complications after congenital cataract surgery without intraocular lens implantation in the first 18 months of life. Am J Ophthalmol. 2008; 146 (1): 1-7.

29. Egbert JE, Wright MM, Dahlhauser KF, Keithahn MA, Letson RD, Summers CG. A prospective study of ocular hypertension and glaucoma after pediatric cataract surgery. Ophthalmology. 1995; 102: $1098-101$.

30. Miyahara S, Amino K, Tanihara H. Glaucoma secondary to pars plana lensectomy for congenital cataract. Graefes Arch Clin Exp Ophthalmol. 2002; 240: 176 -9.

31. Rabiah PK. Frequency and predictors of glaucoma after pediatric cataract surgery. Am J Ophthalmol. 2004; 137: $30-7$.

32. Parks MM, Johnson DA, Reed GW. Long-term visual results and complications in children with aphakia: a function of cataract type. Ophthalmology. 1993; 100: $826-40$.

33. Johnson CP, Keech RV. Prevalence of glaucoma after surgery for PHPV and infantile cataracts. J Pediatr Ophthalmol Strabismus. 1996; 33: $14-7$.

34. Ariturk N, Oge I, Mohajery F, Erkan D, Turkoglu S. Secondary glaucoma after congenital cataract surgery. Int Ophthalmol. 1998; 22: 175-80.

35. Chen TC, Bhatia LS, Halpern EF, Walton DS. Risk factors for the development of aphakic glaucoma after congenital cataract surgery. Trans Am Ophthalmol Soc. 2006; 104: 241-51.

36. Lundvall A, Zetterstrom C. Complications after early surgery for congenital cataracts. Acta Ophthalmol Scand. 1999; 77: 677-80.

37. Vishwanath M, Cheong-Leen R, Taylor D, Russell-Eggitt I, Rahi J. Is early surgery for congenital cataract a risk factor for glaucoma? Br J Ophthalmol. 2004; 88: 905-10.

38. Walton DS. Pediatric aphakic glaucoma: a study of 65 patients. Trans Am Ophthalmol Soc. 1995; 93: 403- 13.

39. Freedman SF, Lynn MJ, Beck AD, Bothun ED, Örge FH, Lambert SR, Infant Aphakia Treatment Study Group. Glaucoma-Related Adverse Events in the First 5 Years After Unilateral Cataract Removal in the Infant Aphakia Treatment Study. JAMA Ophthalmol. 2015; 133 (8): 907-14.

40. Kim KH, Ahn K, Chung ES, Chung TY. Clinical outcomes of surgical techniques in congenital cataracts. Korean J Ophthalmol. 2008 Jun; 22 (2):87-91. doi: 10.3341/kjo.2008.22.2.87.

41. Lambert SR, Buckley EG, Drews-Botsch C, DuBois L, Hartmann E, Lynn MJ, Plager DA, Wilson ME. Infant Aphakia Treatment Study Group. Arch Ophthalmol. 2010; 128 (1): 21-7.

42. Plager DA, Lynn MJ, Buckley EG, Wilson ME, Lambert SR. Complications, Adverse Events, and Additional Intraocular Surgery 1 Year after Cataract Surgery in the Infant Aphakia Treatment Study. Ophthalmology. 2011; 118: 2330-4.

43. Trivedi RH, Wilson ME Jr, Bartholomew LR, Lal G, Peterseim MM. Opacification of the visual axis after cataract surgery and single acrylic intraocular lens implantation in the first year of life. J AAPOS. 2004; 8 (2): 156-64.

44. Negalur M, Sachdeva V, Neriyanuri S, Hasnat Ali M, Kekunnaya R. Long-term outcomes following primary intraocular lens implantation in infants younger than 6 months. Indian J Ophthalmol. 2018; 66 (8): 1088-93. 
45. O'Keefe M, Fenton S, Lanigan B. Visual outcomes and complications of posterior chamber intraocular lens implantation in the first year of life. J Cataract Refract Surg. 2001; 27 (12): 2006-11.

46. Shrestha UD, Shrestha MK. Visual axis opacification in children following paediatric cataract surgery. JNMA J Nepal Med Assoc. 2014; 52: 1024-30.

47. Fenton S, O'Keefe M. Primary posterior capsulorhexis without anterior vitrectomy in pediatric cataract surgery: longer-term outcome. J Cataract Refract Surg. 1999; 25 (6): 763-7.

48. Vasavada AR, Trivedi RH, Singh R. Necessity of vitrectomy when optic capture is performed in children older than 5 years. J Cataract Refract Surg. 2001; 27 (8): 1185-93.

49. Ramsay AL, Diaper CJ, Saba SN, Beirouty ZA, Fawzi HH. Simultaneous bilateral cataract extraction. J Cataract Refract Surg. 1999 Jun; 25 (6): 753-62.

50. Totan Y, Bayramlar H, Yilmaz H. Bilateral paediatric cataract surgery in the same session. Eye (Lond). 2009; 23 (5): 1199-205.

51. Chang DF. Simultaneous bilateral cataract surgery. Br J Ophthalmol. 2003 Mar; 87 (3): 253-4.

52. Chung JK, Park SH, Lee WJ, Lee SJ. Bilateral cataract surgery: a controlled clinical trial. Jpn J Ophthalmol. 2009 Mar; 53 (2): 107-13.

53. Dave H, Phoenix V, Becker ER, Lambert SR. Simultaneous vs sequential bilateral cataract surgery for infants with congenital cataracts: Visual outcomes, adverse events, and economic costs. Arch Ophthalmol. 2010 Aug; 128 (8): 1050-4.

54. Arshinoff SA, Chen SH. Simultaneous bilateral cataract surgery: Financial differences among nations and jurisdictions. J Cataract Refract Surg. 2006; 32 (8): 1355-60.

55. Arshinoff SA, Odorcic S. Same-day sequential cataract surgery. Curr Opin Ophthalmol. 2009; 20 (1): 3-12.

56. Lambert SR, Lynn MJ, Reeves R, Plager DA, Buckley EG, Wilson ME. Is there a latent period for the surgical treatment of children with dense bilateral congenital cataracts? J AAPOS. 2006; 10 (1): 30-6.

57. Lundvall A, Kugelberg U. Outcome after treatment of congenital bilateral cataract. Acta Ophthalmol Scand 2002; 80: 593-7.

58. Robb RM, Petersen RA. Outcome of treatment for bilateral congenital cataracts. Ophthalmic Surg 1992; 23: 650-6.

59. Pandey SK, Wilson ME, Trivedi RH, et al. Pediatric cataract surgery and intraocular lens implantation: current techniques, complications, and management. Int Ophthalmol Clin. 2001; 41 (3): 175-96.

60. O'Keefe M, Fenton S, Lanigan B. Visual outcomes and complications of posterior chamber intraocular lens implantation in the first year of life. J Cataract Refract Surg. 2001; 27 (12): 2006-11.

61. Shrestha UD, Shrestha MK. Visual axis opacification in children following paediatric cataract surgery. JNMA J Nepal Med Assoc. 2014; 52: 1024-30.

62. Cheng KP, Hiles DA, Biglan AW, Pettapiece MC. Visual results after early surgical treatment of unilateral congenital cataracts. Ophthalmology 1991; 98: 903-10.

63. Gregg FM, Parks MM. Stereopsis after congenital monocular cataract extraction. Am J Ophthalmol 1992; 114: 314-7.

64. Whitman MC, Vanderveen DK. Complications of pediatric cataract surgery. Semin Ophthalmol. 2014 SepNov; 29 (5-6): 414-20.

65. Zetterström C, Lundvall A, Kugelberg M. Cataracts in children. J Cataract Refract Surg. 2005; 31 (4): 824-40.

66. Loudon SE, Fronius M, Loman CW, et al. Predictors and a remedy for noncompliance with amblyopia therapy in children measured with the occlusion dose monitor. Invest Ophthalmol Vis Sci. 2006; 47: 4393-400.

67. Norman P, Searle A, Harrad R, Vedhara K. Predicting adherence to eye patching in children with amblyopia: An application of protection motivation theory. Br J Health Psychol. 2003; 8: 67-82.

68. Dixon-Woods M, Awan M, Gottlob I. Why is compliance with occlusion therapty for amblyopia so hard? A qualitative study. Arch Dis Child. 2006; 91: 491-4. 